Alma Mater Studiorum - Università di Bologna DEPARTMENT OF ECONOMICS

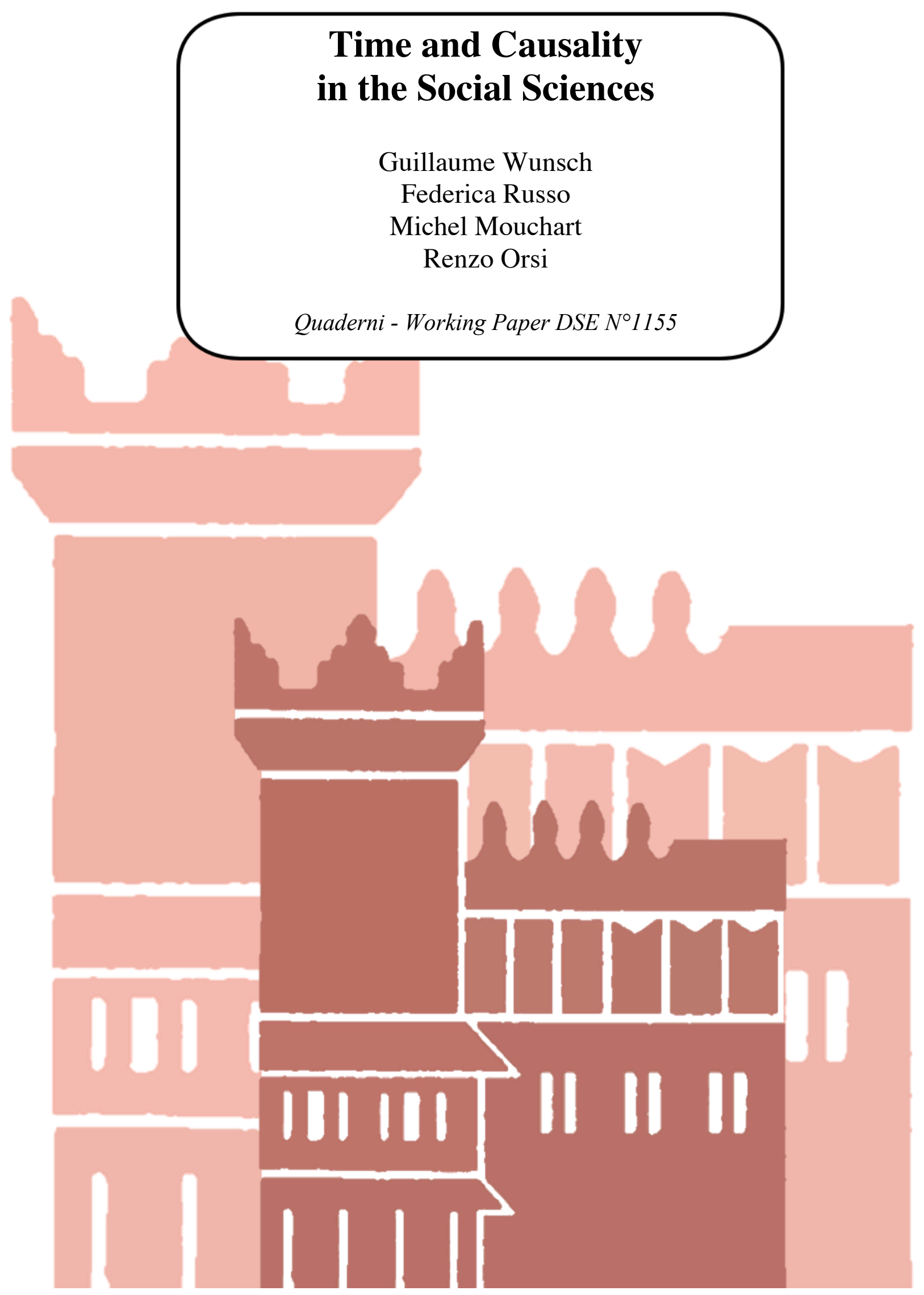




\section{Time and Causality in the Social Sciences}

\section{Guillaume WUNSCH ${ }^{\mathrm{a}}$, Federica RUSSO ${ }^{\mathrm{b}}$, Michel MOUCHART ${ }^{\mathrm{c}}$ and Renzo ORSI ${ }^{\mathrm{d}}$}

a Demography, UCLouvain, Belgium

b Philosophy of Science, University of Amsterdam, The Netherlands

${ }^{\mathrm{c}}$ Institut de Statistique, Biostatistique et Sciences Actuarielles

(ISBA), UCLouvain, Belgium

d Department of Economics, University of Bologna, Italy

September 25, 2020

Corresponding Author: Michel Mouchart, ISBA, Université catholique de Louvain, 20 Voie du Roman Pays, B-1348 Louvain-la-Neuve, Belgium. e-mail: michel.mouchart@uclouvain.be

Aknowledgements. The authors are deeply grateful to Laurent Toulemon and Frans Willekens for their quite interesting comments and suggestions on a previous version of this paper. 


\section{NON TECHNICAL SUMMARY}

This article deals with the role of time in causal models in the social sciences, in contrast to time-free models. More specifically, it aims at taking time into account in structural causal modeling. For this purpose, the paper refers to the important discussion on time and causality in the philosophy of science, and it outlines how time has been taken into account in the practice of social science research, taking demography and economics as examples. As a first basic consideration it must be said that taking into account the role of time in a causal structure is a complex issue. Temporal information is useful to the extent that it is placed in a correct causal structure, and thus further corroborating the causal mechanism or generative process explaining the phenomenon under consideration. The accepted view in the social sciences is that causes should precede their outcomes in time. This is a necessary (but not a sufficient) condition for causality. It is important to note, however, that social science practice has shown that the order in which variables are observed does not necessarily give the correct causal ordering. In other words, in many cases, part of the generative or causal process producing the outcomes remains latent, as many observed events in the social sciences result from a decision-making process depending on the intentions, preferences, expectations, and constraints of the agent or actor. This paper is concerned with how, in a field such as the social sciences, studying the generative process underlying the causal relations, requires a thorough reflection about time. While we fully acknowledge that the temporal ordering of the data that are observed cannot provide ipso facto the causal ordering of variables, we also grant that one cannot escape the fact that causal inference must rely, for better or worse, on the information available. As pointed out by John Hicks in his book Causality in Economics (1980), observational models in the social sciences are embedded in historical time, in contrast to experimental models in the natural sciences that are usually independent of historical time. Social facts are tied to time and place, and are partly the result of the past. In the light of how time can be used in structural models, our suggestion is to think of the relation between cause and effect more in terms of a process, of which we try to understand the key points, moments, actors, etc. This is the main idea behind Structural Causal Modeling in which 'structure' has a meaning that goes beyond 'statistical' structure, and that instead also has to do with 'explanation' or with 'explanatory mechanisms'. In turn, explanatory mechanisms are those for which it is possible 
to offer a causal ordering of the variables that takes time into account. In other words, the causal structure is more important than a mere temporal ordering of the variables. This is because, with due exceptions in which the temporal order of observations corresponds to the real temporal ordering of causes and outcomes, plausibility of a causal structure will contribute more to explaining a phenomenon than the sole temporal ordering of variables as observed. Temporal information is useful only to the extent that it is placed in a correct causal structure, and thus further corroborating the mechanism explaining the phenomenon under consideration. Despite the fact that the causal ordering of variables is more relevant for explanatory purposes than the temporal order, the former should nevertheless take into account the time-patterns of causes and effects, as these are often episodes rather than events. Most of our behaviours actually extend over a period of time, be they causes or effects. For this reason in particular, we believe that time should play a central role in our causal models, more than it has until now. 


\begin{abstract}
This article deals with the role of time in causal models in the social sciences, in particular in structural causal modeling, in contrast to time-free models. The aim is to underline the importance of time-sensitive causal models. For this purpose, it also refers to the important discussion on time and causality in the philosophy of science, and examines how time is taken into account in demography and in economics as examples of social sciences. Temporal information is useful to the extent that it is placed in a correct causal structure, and thus further corroborating the causal mechanism or generative process explaining the phenomenon under consideration. Despite the fact that the causal ordering of variables is more relevant for explanatory purposes than the temporal order, the former should nevertheless take into account the time-patterns of causes and effects, as these are often episodes rather than single events. For this reason in particular, it is time to put time at the core of our causal models.
\end{abstract}

Keywords: Time, Causality, Social Sciences, Demography, Economics, Structural Modeling, Causal Mechanism. 


\section{Contents}

$\begin{array}{llr}1 & \text { Introduction } & 6\end{array}$

2 On Time and Causality $\quad 6$

2.1 Time .................... 6

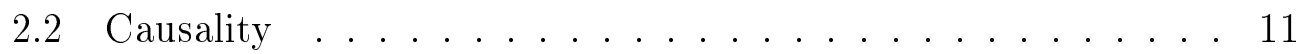

3 Time and Causality in Demography 13

4 Time and Causality in Economics $\quad 16$

5 Time in Structural Causal Modeling 21

$\begin{array}{lll}6 & \text { Conclusions } & 27\end{array}$

$\begin{array}{lll}7 & \text { References } & 29\end{array}$ 
The mystery of time is ultimately, perhaps, more about ourselves than about the cosmos. (Carlo Rovelli)

\section{Introduction}

The above quotation, drawn from Carlo Rovelli's book The Order of Time (2018), reminds us that time is not only a physical quantity but also a personal experience. However, the present paper does not deal with the human experience of time and causality; for this, see for example M.J. Buehner (2014) and the corresponding special issue of the journal Frontiers in Psychology. Nor do we discuss the very particular relation between time and causation in physics; on this topic, see e.g.R. Davidson (2013), and more generally C. Rovelli (op.cit.) on the concept of time.

This article deals instead with the role of time in causal models in the social sciences, in contrast to time-free models. More specifically, it aims at taking time into account in structural causal modeling, such as in the framework developed by Russo, Wunsch and Mouchart (2019). For this purpose, the paper refers to the important discussion on time and causality in the philosophy of science (e.g.Leuridan and Lodewyckx, 2019), and it outlines how time has been taken into account in the practice of social science research, taking demography and economics as examples. This paper shows that taking into account the role of time in a causal structure is a complex issue.

\section{On Time and Causality}

\section{$2.1 \quad$ Time}

From a philosophical perspective, it is interesting to note that a large part of the literature aims to establish a relation between time and causality. Causal relations, unlike correlations, are notoriously asymmetric: $X$ causes $Y$ is not equivalent to $Y$ causes $X$, and typically if $X$ causes $Y$ then $Y$ most often doesn't cause $X$ at the same instant. What is often left implicit in the idea of causal asymmetry is that causes are temporally prior to their effects. What is at stake, in fact, is an intricate relation between causation and time, which has been the object of important contributions from philosophy of science 
and social science methodology. The general strategy, however, has been to take one as primitive, in order to define the other.

For instance, for some philosophers, if we take the flow of time from past to present to future as given, the temporal order of variables will correspond to the causal order. Put in simple terms, this has been the argumentative strategy of Hans Reichenbach (1956), who was effectively trying to deduce causal ordering from the probability of observing events in a certain temporal order, from past to present, rather than in the reverse order. In more recent times, analogue arguments have been offered and revived by Huw Price (1996), who tried to infer the asymmetry of the cause-effect relation from the laws of thermodynamics. In the pioneering work of Patrick Suppes (1970), time ordering of the causes and effects is assumed, and often given by the conceptual framework used to interpret the data. Thus, while time ordering of events is mentioned in the definition of 'prima facie' cause, it is then left implicit in the definitions of different types of cause. So, in Suppes' approach temporal ordering fixes the causal ordering, but within a given conceptual framework to be specified.

The accepted view in the social sciences is that causes should precede their outcomes in time. This is a necessary (but not a sufficient) condition for causality. It is important to note, however, that social science practice has shown that the order in which variables are observed does not necessarily give the correct causal ordering. For example, a change in occupation might follow a change in residence but the latent decision to change one's occupation might precede the decision to migrate. In other words, in many cases, part of the generative or causal process producing the outcomes remains latent, as many observed events in the social sciences result from a decision-making process depending on the intentions, preferences, expectations, and constraints of the agent or actor. These factors may moreover vary over time. In some situations, such as in the case of divorce, the decision is taken by more than one agent. This compounds the difficulty of correctly specifying the latent decision-making process leading to the observed event. Possible nonobservability of some of the variables is a major problem in the development of relevant structural models.

Of course, retrospective questions can to some extent inform us on the decision-making process, but the answers are often influenced by recall lapses and by motivated reasoning, i.e. producing justifications that are most desired instead of the actual argument. For example, an agent might declare that his/her change in occupation was the result of free choice, though in 
fact (s)he was forced to leave his/her former job. One adapts the evidence to justify one's behavior.

Recall lapses are not the only problem in the timing of events. If official registers record births or deaths, for example, according to the day of occurrence, dating can be more imprecise in other sources. For example, in the Belgian Health Interview Survey of 2013, questions on stress and wellbeing relate to the past weeks, with no precise date. In innumerate populations, age itself is often poorly recorded in censuses or surveys, leading to age heaping in population pyramids. In economics, some econometric indicators such as growth rate or inflation are usually recorded at quarterly or monthly scales. Temporal ordering may thus prove problematic for causal ordering. One must clearly distinguish the generative or causal process underlying the ordering of cause-effect relations from the manifestation of this process producing the (possibly inaccurate and incomplete) temporal data that are actually observed. In other words, as stressed a long time ago by Blalock (1968) and more recently Gérard (2006), one must distinguish the conceptual model at the theoretical level from the operational model at the observational level. In many situations, due to lack of data, some of the concepts in the former model cannot be translated into relevant measurable indicators in the latter model. For instance, the whole debate about time series and spurious correlations, see e.g.Reiss (2006), was meant to show that even the cleanest temporal sequence cannot be taken as a foolproof basis for causality.

This paper is concerned with how, in a field such as the social sciences, studying the generative process underlying the causal relations, requires a thorough reflection about time. While we fully acknowledge that the temporal ordering of the data that are observed cannot provide ipso facto the causal ordering of variables, we also grant that one cannot escape the fact that causal inference must rely, for better or worse, on the information available. In the perspective of causal assessment, a thorough evaluation of the quality and relevance of the information at hand must always be performed. In the structural causal modeling perspective of this paper, the terms generative process and causal mechanism are used interchangeably for the ordered network of cause-outcome relations. We adopt here the characterization of 'minimal mechanism' of Glennan and Illari (2017, p.92), because it is broad and liberal enough to include both these terms as well as 'causal process':

"A mechanism for a phenomenon consists of entities (or parts) whose activities and interactions are organized so as to be responsible for the phenomenon." 
For our purposes, the possible differences between these three terms do not matter. What does count is that, in all cases, there is an important temporal dimension that poses theoretical and practical challenges.

This overview on time and its relation to causality shows that the goal in philosophy is often to use one to define the other, as if one should have priority. Such priority may be understood in metaphysical or epistemic terms. For instance, one may argue that the arrow of time is metaphysically prior, and this gives us the arrow of causality. Or, one could consider the flow of time as an 'epistemic' tool to make inferences about causal ordering. However, the following sections show that, in the practice of the social sciences, and notably of economics, reasoning about temporal and causal ordering is not fixed. Or, differently put, the question at stake is not to determine, once and for all, whether temporal priority defines causal priority or vice-versa. Instead, the question is how best to use temporal knowledge, when available, to make causal inference and vice-versa. In other words, what emerges from the practice of the social sciences, is a kind of pragmatic approach to the role of time in causality. Indeed, one can sometimes infer causality from crosssectional data, where temporal information is not reflected in the observed values of the variables. Here, time can be encoded using background and contextual knowledge (Wunsch, Russo, Mouchart, 2010).

One can distinguish, from practice in demographic research (Section 3), three main aspects of time, developed in the following paragraph. In a broader perspective, one could also consider other aspects of time, for example 'natural' time such as the cycle of the seasons and its relation with agricultural production (see Section 4), or the seasonal cycle of religious rules and practices and its impact on the occurrence of marriages and conceptions (Matsuo and Matthijs, 2018). Biological cycles are other examples of "natural' time. For instance, fecundability (the probability of conceiving) varies greatly across the menstrual cycle. Or one can be interested in 'social' time, such as work time, life stages, etc. On this topic, and its relation to natural and individual time, see the interesting paper by van Tienoven (2019). Sequence analysis can be used in this case to examine, for example, the sequential patterns of housing trajectories (Mikolai and Kulu, 2019). Courgeau (2018) has thoroughly discussed the pros and cons of this approach, in comparison with event-history analysis and network analysis.

Consider now three main aspects of time as derived from demographic practice. Firstly, time is at the basis of the ordering of events, such as a change in occupation followed by a change in residence. In the social sci- 
ences, as pointed out above, the temporal priority of the cause over the outcome (the happens-before relation), with an appropriate time-lag, is a necessary (but not sufficient) condition for causality. Following the Russo Williamson thesis (Gillies, 2011), one also needs in addition that the cause make a difference to the outcome. Furthermore, the observed temporal order does not necessarily correspond to the causal order, for reasons given above. Secondly, the passage of time is associated with the ageing and decline of biological organisms, such as human beings, or the wearing out of manmade objects, such as your car. Thirdly, and more specifically for the social sciences and humanities, history is entrenched in time. Events, such as 'Black Tuesday' (October 29, 1929) and the stock market crash, and institutions, such as the Central Bank and its activities, are specific to a historical time and context. The latter have to be taken into account in causal assessment.

As pointed out by John Hicks in his book Causality in Economics (1980), observational models in the social sciences are embedded in historical time, in contrast to experimental models in the natural sciences that are usually independent of historical time. Social facts are tied to time and place, and are partly the result of the past. This explains, for example, why most time-series in the social sciences are non-stationary, and why causal models in the social sciences are time- and context-dependent, and are specific to a reference population that has to be circumscribed (Russo, Wunsch and Mouchart, 2019).

Sections 3 and 4 will shed some light on these various aspects of time and their relation to causal processes, focusing in particular on two specific disciplines within the social sciences, demography (Section 3) and economics (section 4). In demography, the three main aspects of time considered above are at the very basis of causality assessment, while in economics concepts such as rationality of the economic agents or opportunity cost, are usually considered independent of historical time. Theories and models in economics would thus be closer, to some extent, to those in the natural sciences than to the theories and models developed in demography or in anthropology, to give another example. The issue of time is, of course, also addressed in the other social sciences, such as sociology. See, in particular, the literature review on the sociology of time in Bergmann (1992). 


\subsection{Causality}

The concept of causality is also much discussed in philosophy and in science. There is no unanimous definition of causality able to capture the meaning and use of the concept across different scientific domains. While, philosophically speaking, pluralist approaches such as the one developed by Illari and Russo (2014) are gaining traction and eventually prove a useful approach, we confine the discussion here to some specific uses of 'cause', 'causality', 'causation', from the literature on causal modeling in quantitative social science research.

In the context of quantitative modeling, causality has to do, prima facie, with how variables are correlated. These models often follow the 'causation via association approach', as D.R. Cox (1992) has called it, i.e. a variable $\mathrm{X}$ is a plausible cause of another variable $\mathrm{Y}$ if the dependence between the two cannot be eliminated by introducing additional variables in the analysis. The general question behind different modeling practices is how changes in the putative cause $\mathrm{X}$ are related to changes in the putative effect(s) Y, and what would possibly ground such correlation (Russo, 2009). Asking what 'would possibly ground a correlation' amounts to answering 'explanationseeking why-questions' (dixit Carl Hempel). For this purpose, one needs to go beyond the 'causation as robust dependence' approach in favor of what H.P. Blossfeld (2009) has coined a 'causation as generative process' approach. One should characterize the properties of the underlying data generating process, i.e. the mechanism behind the data.

For this reason, causal assessment is based, in the present paper, on structural causal modeling (SCM), the characteristics of which are the following (Russo, Wunsch and Mouchart, 2019). Firstly, a recursive decomposition of the joint distribution of the variables, based on an ordered sequence of intelligible sub-mechanisms (entities and activities), reflecting the causal ordering of the variables underlying the putative mechanism generating the data. Each of the sub-mechanisms is time-dependent, as causes precede their effects in time. Secondly, congruence with background knowledge, concerning the context and period, the causal ordering and the role-function of the variables. Thirdly, invariance of the recursive decomposition for a specified population and historical context.

Though SCM focuses on the individual level, agents/actors are of course part of social networks of interacting individuals, and are influenced by higher-level units of analysis. A causal explanation should also take these 
multilevel interrelations into account. For example, scientists collaborate with others, and these networks are embedded in disciplinary and organizational levels. This relational structure of scientific collaboration plays a role in the scholars' success, i.e. the funding they receive (Bellotti et al., 2016).

In the context of econometrics, causality has sometimes been understood in a rather different way. Take, for example, the so-called Granger-causality in econometrics. Actually, Granger-causality does not correspond to a classic definition of causality (see e.g. Little (2011) from a causal realist perspective) but to a concept of 'self-predictivity', in the sense of sufficiency for prediction (Florens, Mouchart and Rolin 1990, p.255). Granger-causality refers in fact to a concept of non-causality: $\mathrm{X}$ does not cause $\mathrm{Y}$ if the earlier values of $\mathrm{X}$ have no impact on the later values of $\mathrm{Y}$. Therefore, it does not describe a structural cause and effect relationship. Following is another example of a view on causality that differs from the generative process approach. In Big Data-driven analysis using data mining techniques, Big Data being characterized both by many data and many variables, causality usually means uncovering hidden associations or statistical dependencies among variables. These can serve for descriptive purposes, for finding unsuspected patterns in the data that may possibly lead to discovering novel mechanisms, or for automatic detection in the data of outcomes of known causes. The focus is always on extracting strong relationships or patterns among variables from the data. See Hassani, Huang, and Ghodsi (2018) for a thorough overview of data mining techniques applied to Big Data analysis. While this approach is rather widespread, Big Data analyses could also be carried out in a structural causal modeling perspective, but this issue lies outside the scope of the present paper.

The rest of this paper is structured as follows. Section 3 examines the reference to the three main aspects of time in demographic research, as an example of a social science dealing with time. As another example, Section 4 is devoted to the role of time in economic theories and models. Section 5 examines, in a generative process perspective, how time can be incorporated into structural causal models, considering among others that causes and outcomes can be events or episodes. It points out some practical difficulties in integrating time in structural causal models. Finally, Section 6 concludes that the causal ordering of variables is more relevant for explanatory purposes than the temporal ordering. 


\section{Time and Causality in Demography}

For a broader perspective on demography, the reader is referred to Daniel Courgeau (2007) who has thoroughly examined the change in the paradigms of demographic research, from the macro to the micro level, and then to multilevel analysis. Frans Willekens has also been a pioneer in building a bridge between the micro and macro levels with his MicMac demographic models (Willekens, 2005).

Since its origin in the 17th century, demography as a science has been concerned with time. One of its founding fathers, John Graunt, was instrumental in computing the first life table taking broad age-intervals into account (Graunt, 1662), based upon the Bills of Mortality, i.e. weekly data on burials in London. Actually, the Bills did not record age at death. The age-intervals used by Graunt are largely conjectural. For a recent overview of Graunt's achievements, see Harkness (2020). Age is, of course, the time elapsed since birth and it is a crucial factor associated with the risk of dying, as frailty usually increases with ageing. A recent example is the age-effect in the risk of dying from COVID-19. The mean age at death, also called the expectation of life at birth, is a convenient summary indicator of the life table. Life tables by age (and sex) became one of the main tools in the demographic arsenal, and the framework was eventually adapted to the study of fertility by age, among others. As one knows, age is an important factor associated with fertility too. From the age-specific fertility rates, one can derive in particular the mean age at childbearing and the average number of children per woman. Usually, these tables were computed for a specific period, e.g.for the study of mortality or fertility by age in the period 1980-84 for example, using the so-called fictitious (or synthetic) cohort approach (see Wunsch and Termote, 1978). Time trends could then be examined period by period, and discussed in a causal perspective by taking into account the historical contexts of the periods considered.

With the increasing availability of long series of vital statistics, and thanks to the impetus given by such demographers as Norman Ryder in the US or Louis Henry in France, the focus of analysis gradually changed from period to cohort. The latter stands for a group of persons experiencing the same eventorigin (birth, marriage, occurrence of the third child, etc.) during the same year or time-period. As Ryder (1965, p. 844) has pointed out, each cohort has a unique location in the stream of history. Thus, each cohort is differentiated from all others. Fertility rates, for example, were now examined by birth 
cohort and age; divorce was studied by marriage cohort according to the time passed since marriage, the probability of having a fourth child computed by parity cohort according to the duration elapsed since the birth of the third child, etc., and cohort- and duration-effects were often highlighted. In all these cases, duration is measured according to some arbitrary units of time, such as year, month, etc., that act as points of reference. The choice of the unit has an impact on the analysis of durations. The data and indicators remain however at the aggregate level, as vital statistics are not published at the individual level. A useful visual device for presenting results by age, period, and cohort, dating from the end of the 19th century, has been the socalled Lexis diagram (see Vandeschrick, 2001). Of course, the three variables are linearly related. Without further constraints, the separate influence of each of these three variables (age, period, cohort) cannot be identified.

With the advent of computers, a further step could now be taken: the analysis of longitudinal individual data, as provided by retrospective questions at censuses and especially at surveys. A major advantage of longitudinal individual data is that it enables the temporal ordering of possible causes and outcomes. To give an example in the field of fertility, Laurent Toulemon (2006) has studied the relation between age at first birth and number of children born, using the individual data provided by the French Family History Survey of 1999, taking in particular into account the woman's year of birth and her level of education. He has then compared his individual-level results to the macro situations and trends among the European countries and the US. Toulemon shows that at the micro level the number of children born per woman in France does depend on the age at first birth, even when educational level is controlled. The relation is not observed however at the country or macro level in an international comparison, and it varies across cohorts and time. Of course, temporal order does not necessarily imply causal order, though causes must precede their effects in time. From a survey on migration histories, for example, one can see if a change in residence has followed a change in occupation, or vice-versa, though - as previously discussed - surveys rarely record the decision-making process that has led to these events.

Demographers can also adopt a life course approach, when studying the timing, i.e. the ordering, of successive births during one's childbearing period or the series of changes of residence one has made during one's lifetime. For an interesting presentation of the life course approach, stressing in particular the interdependencies across time, domains, and levels, see Bernardi, Huinink and Settersten (2019). One can relate individual fertility or mi- 
gration, or more generally individual behavior, to one's socio-economic and cultural characteristics and contexts, and their interdependencies, such as ethnicity, social background, childhood experiences, educational level, and to the social transitions and sequences of events experienced during one's life span. The choice of these explanatory variables is of course dependent upon the time-period and context one is considering, i.e. the historical time during which the events are occurring. For example, the determinants of fertility in high-fertility Niger are substantially different from those in low-fertility Germany. Furthermore, the approach is quite demanding in terms of relevant data, both micro and macro, their interrelations, and their change over time. Another problem is the great heterogeneity in life courses, often requiring the use of dimensionality-reduction methods, such as latent class growth models, as the number of individuals is usually small but the number of variables is large.

Obviously, the retrospective approach is not suited for the analysis of mortality: "dead men tell no tales", as the saying goes! More or less at the same time however, epidemiologists could study morbidity and mortality using individual prospective data, such as those provided by the Framingham Heart Study (from 1950 onwards). Prospective follow-up birth-cohort studies are currently used in demography to examine for example child development over time. Field sites or population laboratories in the developing countries, such as the Matlab project established in 1966 in Bangladesh, have also yielded individual longitudinal data for the study of $i . a$ mortality trends and patterns (Razzaque et al., 2009). Demographers have furthermore benefitted from the development of statistical methods for the analysis of longitudinal data, such as the Cox proportional hazards model and other survival models for event history analysis (see e.g.Florens, Fougère and Mouchart, 2008).

A more recent development has been the spread of record linkage among data sources, with the adoption of a common personal identification number (PIN) in the various sources. As discussed in Wunsch and Gourbin (2018), the same individual may, for example, be interviewed in a health survey and later on recorded in a cancer register, with information collected by the health insurance system and medical statistics, and end up with his/her cause of death specified on a death certificate and a possible autopsy report. Being able to link these individual records together over time, thanks to a PIN, can give the event history or biography of the person in the areas of health, morbidity and mortality. If the observation period is sufficiently long, one can, for example, examine the shift from good health to ill health, 
then to chronic disease, disability and finally death. Owing to currently available registers in several countries, one can also take into account various socio-economic characteristics of the individuals (such as employment), some aspects of their behavior (such as the consumption of medicinal drugs), and their change over time. This linked information can possibly be used to develop relevant structural causal models in the health field. Demographers are currently linking census data with vital statistics for mortality studies, and the Nordic countries among others are using linked registers for the study of health and disease. For example, Oksuzyan et al. (2009) have linked three Danish population-based surveys to three national registers to examine the health-survival paradox, i.e. the fact that women live longer than men, but men often report better health than women. With the powerful computers now available, the record linkage issue is not technical but ethical: how do we guarantee the privacy and absence of disclosure of the data while at the same time using them for scientific purposes?

\section{Time and Causality in Economics}

Very often, in the social sciences, we cannot obtain a complete knowledge of how decisions are formed, since we do not have a thorough and detailed information on the functioning of the system itself and on the context in which the decisions are made. In the analysis of social phenomena, we then proceed by models. Their purpose is to provide a useful construct that allows us to interpret the phenomena of the real world according to a hypothetical structure suggested by theory, and in this interpretation time can play a fundamental role. More generally, regarding the importance of time in economics, the reader is referred, for instance, to two issues of the journal Oeconomia (2017, issues 7- 1 and 7-2).

Time is the dimension in which the passing of events is conceived and measured. It is basically a human construct designed for organization and coordination purposes, and it induces the distinction between past, present, and future. The time factor cannot be ignored if we want to highlight the relevance of the evolutionary aspects of an economic process. For economic time series data, time is unidirectional by construction, since its direction is defined as that of increasing time. In economics, time is quite different from the spatial dimension where we can move spatially back and forth, while there is no time reversibility for empirical observations of time series of eco- 
nomic data. And it is precisely because we can count on the directionality of time in economic time series that it is possible to consider time as a vehicle of causality, for those phenomena for which the causal relationship is supported by economic theory. Taking time into account in economics has given rise to an interesting debate which has resulted in a series of proposals in the various areas of economics, from macroeconomics to microeconomics, both from a theoretical and an empirical point of view. A first important result of this debate was to ascertain that time preferences may vary according to socio-economic status, culture, and levels of development, with the consequences that this entails on the inter-temporal choices of individuals. Another important result is in the area of economic theory dealing with choices in conditions of uncertainty based on expected utility. In this case, the matter of time consistency has brought about an interesting discussion leading to a more descriptively accurate and psychologically grounded representation of time.

The concept of interest rate, which differs according to whether it is defined in the short term or in the long term, implicitly attributes the reason for this difference to the time factor. In fact, time takes on the power to change the value of money according to the so-called discounted cash flow (Kruschwitz and Loeffler, 2005). Moreover, modern economic theories have introduced the concept of time-based competition (Stalk and Thomas, 1990), a kind of strategic game on the markets where the one who arrives first wins. In financial markets, the measurement of the volatility of financial time-series is an important element that is considered for the evaluation and analysis of financial risk. In performing risk management, it is necessary to have a good understanding of how volatility changes over time. The usual approaches to the study of this phenomenon are mainly based on physical time as a persistent measurement of the intervals at which price returns are calculated. These are observed in a price time-series showing variations ranging from minutes to hourly, daily or weekly changes, in which the flow of time is discontinuous. Because of this, it is difficult and inefficient to observe price changes through the use of physical time, since significant patterns of trading risk may be ignored when considering data based on physical time (e.g.weekly, daily, or hourly changes). The importance of trading activity actually depends on the time of the day and on the day of the week (i.e. on a precise instant of time), and physical time fails to represent the full activity of price variations. This is typically a problem of time-aggregation, discussed more fully in Wunsch, Mouchart and Russo (2018). Moreover the time- 
aggregation for a causal event may be different from the time-aggregation for an outcome event.

For many economic phenomena, the observed data are obtained by applying temporal aggregation to the original processes, and this can make it difficult to discover the underlying causal relationships. For example, some econometric indicators, such as growth rate or inflation, are usually recorded at quarterly or monthly scales. Causal interactions between financial assets, however, may take place at weekly or fortnightly scales. One should however note, in the financial field, that the BEDOFIH database collects stock market data in nearly real time (see Eurofidai website). We can say that there is an optimal time frequency for analyzing economic data, that is, the frequency that allows us to grasp in the best possible way the causal relationships underlying the phenomenon under study. Thus, for the analysis of financial markets it is necessary to have high frequency data, as decisions on these markets are made very quickly. For the analysis of other economic phenomena, data can be used instead at a lower frequency, as decisions are made in more extended times.

Another important role of the time factor in economics is given by the principle called the time value of money. It is based on the assumption that rational investors prefer to receive money today rather than the same amount of money in the future, due to the money's potential growth in value over a given period of time. Given that money can earn compound interest, it is more valuable in the present rather than the future. The time value of money is simply given by the basic, or risk free, interest rate. As a general rule, money does not reduce its value unless inflation occurs. Due to the fact that money has the characteristic of usually not losing value, interest is required when it is lent.

This rule lays the foundation for determining the discount rate that should be expected for future cash flows. The same principle, considered mainly for financial assets, is also used to calculate the depreciation of other types of assets. On the basis of this methodology, the intrinsic value of a good is determined, i.e. the value of owning that good for its entire duration. Every product loses its value over time. For example, no one will want to buy yesterday's newspaper today, as perishable products will deteriorate increasingly and therefore lose value with the passage of time. The monetary value of an asset decreases over time due to use, usury, or obsolescence or, ultimately, to unfavorable market conditions. The duration during which the value of a product depreciates is measured by time. 
The fact that the variance of asset returns tends to change over time greatly complicates the analysis. Methods based on stochastic volatility have been developed in the statistical sphere, while other methods designed to evaluate the size of the volatility are mostly based on Fourier analysis and involve considerable computing. To overcome the difficulties of estimating volatility, there is a need to develop new methods able to describe the evolutionary dynamics of the series of prices regardless of price flow over time. Alternative methods have been proposed in the literature and among them a reliable solution is provided by the method based on the use of intrinsic time. Intrinsic time consists in the rescaling of the data, especially the high frequency ones in the financial sector, which - instead of being reported according to calendar time (by day, week, month, quarter etc.) - are expressed in a time dimension that has the aim of capturing the core of the phenomenon (Muller et al., 1993). This new time dimension transforms time in accordance with the intrinsic characteristics of the system being analyzed, providing data with a higher frequency during turbulent periods and a lower frequency during periods of calm. The series of data represented in this way is much more informative on the volatility (risk) of the phenomenon, unlike the series observed according to the standard calendar which, by construction, are unable to capture this type of information.

As seen before, the physical time method is based on a fixed interval point system. Intrinsic time is based on an event system, i.e. the time interval is defined by events. Consequently, while physical time is homogenous, since observations are equally spaced on any chosen time scale, intrinsic time is not homogenous in time. The observation takes place only when the event occurs, independently of the notion of physical time. To define an event according to the system of intrinsic time, a threshold is fixed and any change in price between two local extreme values exceeding this threshold identifies an event. This change in price may be downwards or upwards, and the notion of directional change has been introduced in the literature (Aloud et al., 2012). The intrinsic time concept and related directional change have no relation with the speed of price change. The characteristic of directional change of intrinsic time sets the foundations for a new variance estimator completely detached from time series data observed in equidistant time-periods. Directional change events, both upward and downward, tend to highlight significant periodic changes in the time-series where the magnitude of an event is decided by the researcher. In a completely different way, in physical time, one divides time into periods of equal length, giving rise to the possibility 
of omitting significant events. With intrinsic time, the significant events are highlighted, showing a temporal evolution of prices that is useful for evaluating the dynamic behavior of a financial market and the analysis of the volatility of financial time-series.

These considerations point out the importance of the time factor in economics and its diversified use, but they do not clearly highlight the importance of time for causal assessment. Considering that a main concern of economics is to understand the functioning of the economy, of what is observed day by day, and the functioning of the mechanisms of an economic system, then the concept of time as a background to causal relationships is somehow recovered. However, due to a lack of a detailed knowledge of how the economic world works, and of the functioning of economic mechanisms, economics, like many other disciplines, makes use of theory and models that should help in describing the economic mechanisms. Referring to theory means providing a putative explanation of the phenomenon, i.e. proposing a causal mechanism that contributes to a plausible understanding of the observed reality.

In economics, time is also used to describe the seasonality of certain phenomena. Seasonality is a phenomenon linked to time, which occurs within one year and is caused by changes in weather, and in the calendar and timing of decisions. In agriculture, for example, the seasonality of crop production entails complications in the economic decisions of the producer. In agriculture, investments are made well in advance of production, the latter being uncertain in quantity and quality, as the agricultural production cycle depends considerably on rainfall and more generally on weather conditions which are not foreseeable so far in advance, i.e. when making decisions about which crops to plant. In other words, farmers have to face long delays between the time they spend and their revenue, which also makes access to credit difficult. All this has as a consequence that the realizations and expectations on the prices of agricultural products are uncertain and vary over time, as they depend in an important way on weather trends and on seasonality. In addition, farmers must devote their time to multiple activities distributed over time and according to a temporal schedule that cannot be modified. The preparation of the soil must take place before seeding which follows the use of fertilizers or other treatments, activities that take place before the harvest, followed by the subsequent processing of the product. This temporal schedule is given by the nature of the phenomenon. It follows that when farmers have to cope with anomalous seasonal trends, these anomalies will have to be 
absorbed in the context of their work organization with appropriate adaptations. Despite this, due to the long delays between action and results, farmers will never be able to eliminate the risk of temporal inconsistencies in decisions that may have an impact on productivity and income.

Therefore, always with reference to time in agriculture, an important aspect regards the delays and the mismatches in the various stages of the production process. For example, a delay in seeding due to adverse weather conditions can compromise the production and harvest of the whole year. In fact, the seeding times cannot be extended beyond a certain period, and therefore the interventions that must be carried out before seeding risk not being able to be carried out, with serious consequences on the production cycle and on the results of the entire harvest. In developing economies, the time pattern of the agricultural cycle entails a cycle in sales and consequently in the nutrition of the local population, because there is often no possibility of product storage. This leads to a high sale at the time of the harvest at low prices, while in subsequent periods prices increase and consumption decreases. Thus, a seasonality in consumption is observed which in turn induces a seasonality in the nutritional intake of the resident population.

It is an open problem to understand how farmers can cope with seasonal crop fluctuations in developing countries. It is quite evident that these phenomena push local populations to abandon their native places and move to other areas where they hope to improve their living conditions. The study of these phenomena requires being able to have data on a seasonal basis rather than on an annual basis. Ultimately, seasonality and temporal uncertainty make economic decisions in agriculture more difficult than, for example, in the industrial sector.

\section{Time in Structural Causal Modeling}

As pointed out in Section 2, in this paper causality is assessed in a structural modeling perspective. This section examines some specific problems when analyzing the role of time in structural causal modeling and should be viewed as complementary to the preceding sections where other approaches are also considered.

As summarized in Section 2.2, this approach is based on a recursive decomposition of the joint distribution of the variables, interpretable as an ordered sequence of intelligible sub-mechanisms (entities and activities), re- 
flecting a causal ordering of the variables underlying the putative mechanism generating the data. In this framework, time is implicit in the causal ordering of the variables reflecting the underlying mechanism and sub-mechanisms. The decomposition can be represented by a directed acyclic graph or DAG (Pearl, 2000), though a DAG cannot embody all aspects of the causal structure among variables. In other words, in the present framework, a DAG represents the conditioning structure of a joint distribution, underlying a recursive decomposition, but does not fully provide the properties of the conditional distributions and, therefore, exhibits the mechanisms only partially. The recursive decomposition is based on background knowledge, including the historical context and period, the causal ordering and the role-function of the variables. Finally, considering as structural a mechanism underlying the workings of a data generating process requires that the model enjoy suitable properties of stability, or invariance, under a 'reasonable' class of interventions and of modifications of the environment, i.e. relatively to an underlying population of reference. Indeed, a model that would be different for, say, each observation should not be considered as structural.

Once the vector of variables $X$ is decomposed into an ordered sequence of $p$ components, namely $X=\left(X_{1}, X_{2}, \cdots X_{p}\right)$ (with $p$ typically much larger than 2), a recursive decomposition is a systematic marginal-conditional decomposition of the joint distribution of $X$, namely:

$$
\begin{aligned}
p_{X}(x \mid \theta)= & p_{X_{p} \mid X_{1}, X_{2}, \cdots X_{p-1}}\left(x_{p} \mid x_{1}, x_{2}, \cdots x_{p-1}, \theta_{p \mid 1, \cdots p-1}\right) \\
& \cdot p_{X_{p-1} \mid X_{1}, X_{2}, \cdots X_{p-2}}\left(x_{p-1} \mid x_{1}, x_{2}, \cdots x_{p-2}, \theta_{p-1 \mid 1, \cdots p-2}\right) \cdots \\
& \cdot p_{X_{j} \mid X_{1}, X_{2}, \cdots X_{j-1}}\left(x_{j} \mid x_{1}, x_{2}, \cdots x_{j-1}, \theta_{j \mid 1, \cdots j-1}\right) \cdots p_{X_{1}}\left(x_{1} \mid \theta_{1}\right)
\end{aligned}
$$

where each $\theta_{j \mid 1, \cdots j-1}$ stands for the parameters characterizing the corresponding conditional distribution $p_{X_{j} \mid X_{1}, X_{2}, \cdots X_{j-1}}$. For more details, see Mouchart, Russo and Wunsch (2010).

The purpose of this section is to examine how various aspects of time can be taken into account in structural causal modeling. In particular, a cause or an outcome can be considered as an event, such as a change in occupation at time $\mathrm{t}$ leading to a change in residence at time $\mathrm{t}+\mathrm{k}(\mathrm{k}>0)$. But a cause or an outcome can also be an episode spread over a period of time t to $t+n$, for instance an unhealthy diet leading to the progressive development of atherosclerosis. In structural modeling, this issue does not seem to be much discussed 
but there are exceptions in epidemiology, such as marginal structural models with time-dependent treatments and time-dependent confounders, or models taking into account time-dependent treatments and moderators.

In demography, three time-effects have been distinguished, corresponding to three main aspects of time outlined in Section 2: duration effects, period effects, and cohort effects. For example, an age-specific risk of dying is dependent upon age (the more one ages, the higher the risk), upon the period (there are good and bad years for the flue), and upon the cohort (due to cohort selection effects over time). Compared with demography, in economics - particularly in the financial environment - the role of time may be quite heterogenous not only among the financial agents but also in historical time. This puts into perspective the distinction drawn in Section 4 between physical and intrinsic time. Furthermore, as stressed in particular by Kelly and McGrath (1988), causes and outcomes can present temporal features that should be taken into account. For example, the cause and outcome processes can have different temporal shapes (e.g.linear, delayed, cyclical, etc.) that have to be considered in the causal model.

A first problem to be considered is the feedback effect. It was said in Section 2 that if $X$ causes $Y, Y$ does not cause $X$. If time is taken into account, and if a suitable mechanism is put forward, this restriction can be waived: $X$ causes $Y$ during a first period of time and $Y$ causes $X$ during a second period of time. More formally, a feedback effect may be represented by the following DAG under further conditions:

$$
X_{t_{1}} \rightarrow Y_{t_{2}} \rightarrow X_{t_{3}} \quad X_{t_{3}} \Perp X_{t_{1}} \mid Y_{t_{2}} \quad t_{1}<t_{2}<t_{3}
$$

with the corresponding recursive decomposition:

$$
p_{X_{t_{1}}, Y_{t_{2}}, X_{t_{3}}}=p_{X_{t_{1}}} \cdot p_{Y_{t_{2}} \mid X_{t_{1}}} \cdot p_{X_{t_{3}} \mid Y_{t_{2}}}
$$

The differences $t_{3}-t_{2}$ and $t_{2}-t_{1}$ represent the time-lags between the occurence of the cause and that of the effect. For the sake of simplification, the parameters have not been explicitly written in the previous equation. This example shows that it is necessary to specify time information about the working of the mechanism. In some cases, and following also Suppes (1970), time information is provided by background knowledge or available theories, and structural modeling can, in such cases, avoid explicit specification of time parameters. 
A second problem to be considered deals with the causal DAG $X \rightarrow Y$ where $X$ is a function of time between $t_{1}$ and $t_{2}$ and where $Y$ is a function of time between $t_{3}$ and $t_{4}$, with $t_{1}<t_{2}<t_{3}<t_{4}$. The corresponding recursive decomposition can then be written:

$$
p_{X_{t_{1}}, X_{t_{2}}, Y_{t_{3}}, Y_{t_{4}}}=p_{X_{t_{1}}} \cdot p_{X_{t_{2}} \mid X_{t_{1}}} \cdot p_{Y_{t_{3}} \mid X_{t_{1}}, X_{t_{2}}} \cdot p_{Y_{t_{4}} \mid X_{t_{1}}, X_{t_{2}}, Y_{t_{3}}}
$$

along with

$$
Y_{t_{3}} \Perp X_{t_{1}}, X_{t_{2}}\left|X_{t_{2}}-X_{t_{1}} \quad Y_{t_{4}} \Perp X_{t_{1}}, X_{t_{2}}\right| X_{t_{2}}-X_{t_{1}}, Y_{t_{3}}
$$

These two equations (4) and (5) represent different cases which have to be made more explicit in specific situations. For instance, the variables $X_{t}$ and/or $Y_{t}$ may change progressively within the intervals $\left[t_{1}, t_{2}\right]$ or $\left[t_{3}, t_{4}\right]$ or may have an evolution in time with an abrupt change between $t_{1}$ and $t_{2}$ or between $t_{3}$ and $t_{4}$ (see Blossfeld, 2009).

In some situations, variable $Y_{t}$ could start changing at the same time as the end of the change in $X_{t}$. In this case, one would have: $t_{1}<t_{2}<t_{3}$ with:

$$
p_{X_{t_{1}}, X_{t_{2}}, Y_{t_{2}}, Y_{t_{3}}}=p_{X_{t_{1}}} \cdot p_{X_{t_{2}} \mid X_{t_{1}}} \cdot p_{Y_{t_{2}} \mid X_{t_{1}}, X_{t_{2}}} \cdot p_{Y_{t_{3}} \mid X_{t_{1}}, X_{t_{2}}, Y_{t_{2}}}
$$

along with

$$
Y_{t_{2}} \Perp X_{t_{1}}, X_{t_{2}}\left|X_{t_{2}}-X_{t_{1}} \quad Y_{t_{3}} \Perp X_{t_{1}}, X_{t_{2}}\right| X_{t_{2}}-X_{t_{1}}, Y_{t_{2}}
$$

In another situation, $Y_{t}$ could start changing before the end of the change in $X_{t}$, e.g.a change in atherosclerosis linked to a change in diet. In this case, one would still have: $t_{1}<t_{2}<t_{3}<t_{4}$ but with:

$p_{X_{t_{1}}, X_{t_{2}}, Y_{t_{2}}, X_{t_{3}}, Y_{t_{4}}}=p_{X_{t_{1}}} \cdot p_{X_{t_{2}} \mid X_{t_{1}}} \cdot p_{Y_{t_{2}} \mid X_{t_{1}}, X_{t_{2}}} \cdot p_{X_{t_{3}} \mid X_{t_{1}}, X_{t_{2}}, Y_{t_{2}}} \cdot p_{Y_{t_{4}} \mid X_{t_{1}}, X_{t_{2}}, X_{t_{3}}, Y_{t_{2}}}$

This situation is more complex. If a feedback mechanism is excluded, one can assume:

$$
X_{t_{3}} \Perp Y_{t_{2}} \mid X_{t_{1}}, X_{t_{2}}
$$

Similarly to the previous cases, one can also assume:

$$
Y_{t_{2}} \Perp X_{t_{1}}, X_{t_{2}}\left|X_{t_{2}}-X_{t_{1}} \quad Y_{t_{4}} \Perp X_{t_{1}}, X_{t_{2}}\right| X_{t_{3}}-X_{t_{1}}, Y_{t_{2}}, X_{t_{3}}
$$

In all the above models, it is assumed that the time points are given and known, and that there are no exogenous variables acting as confounders. 
The previous discussion shows that incorporating time in a causal model may raise difficult modeling issues, especially when there are many timedependent variables.

The econometric literature also considers time-dependent autoregressive models of the type:

$$
X_{t_{1}} \rightarrow X_{t_{2}} \rightarrow X_{t_{3}} \quad X_{t_{3}} \Perp X_{t_{1}} \mid X_{t_{2}} \quad t_{1}<t_{2}<t_{3}
$$

with the corresponding recursive decomposition:

$$
p_{X_{t_{1}}, X_{t_{2}}, X_{t_{3}}}=p_{X_{t_{1}}} \cdot p_{X_{t_{2}} \mid X_{t_{1}}} \cdot p_{X_{t_{3}} \mid X_{t_{2}}}
$$

Equations (11) and (12) give to $X_{t_{2}}$ a role of sufficient statistic with respect to the past of $X_{t}$ but these two equations do not represent, most likely, an adequate explanatory mechanism. For example, one's change in weight partly depends upon one's prior weight but also upon other variables such as diet and various psychological and biological mechanisms. However, autoregressive models may be useful for predictive purposes instead of explanatory purposes. Another self-predictive temporal approach is Granger-causality, briefly discussed in Section 2.2. Here again the model lacks an explicit explanatory mechanism, though it can be useful for predictive purposes.

The econometric literature also considers distributed lag models where the effect of a causing variable is realized according to some form of temporal history of that variable. For instance, consider the joint distribution of 3 time-consecutive variables:

$$
p_{X_{t-2}, X_{t-1}, X_{t}}=p_{X_{t-2}} \cdot p_{X_{t-1} \mid X_{t-2}} \cdot p_{X_{t} \mid X_{t-1}, X_{t-2}}
$$

Here, a mechanism could be represented by the distribution $p_{X_{t} \mid X_{t-1}, X_{t-2}}$. If $X_{t-1}$ and $X_{t-2}$ are related, $X_{t-2}$ however confounds the effect of $X_{t-1}$ on $X_{t}$, as $X_{t-2}$ is, in this case, a common cause of $X_{t-1}$ and $X_{t}$. Once again, an explanatory mechanism should be proposed and tested if one wishes to use these models for causal inference.

Another perspective on time-dependent causal models has been given by Odd Aalen et al. (2012). In that paper, the authors discuss the issue of time dynamics in causal modelling, from a mechanistic point of view similar to the one taken in structural causal modelling. After having examined the problem of association among time-dependent processes, based on the concept of local independence (closely related to Granger-causality), the authors develop a 
dynamic path analysis approach. The latter is an extension of classical path analysis, developed in the 1920s by Sewall Wright in population genetics, with time-dependent variables and an outcome considered as a stochastic process. This methodology has recourse to dynamic path diagrams, i.e. sets of DAGs indexed by time. A more formal presentation is given in Fosen et al. (2006), where the method is applied to a study of survival from liver cirrhosis.

Dynamic path diagrams are time-localised, and path coefficients may change over time, outcomes being increments (or changes over time) in a stochastic process. Rather than time-fixing the values of the variables, the dynamic path diagrams evolve over time giving a sequence of dynamic path models, the set of diagrams being a stochastic process. In a nutshell, the model relates the differential of the outcome process at each point of time $t$ (e.g., when data are collected) denoted by $\mathrm{dY}(\mathrm{t})$, to the covariate processes representing the past influencing $d Y(t)$. The method is especially useful for studying direct and indirect effects in event history and survival analyses. In that paper, the approach is applied for examining the impact of HIV-treatment, by running regressions every month since treatment start on increments in viral loads HIV-1 RNA and CD4 cell counts (an indicator of the reaction of the immune system), the independent variables being lagged values of HIV-1 RNA and CD4 cell counts. Results are then compared for the treatment group versus alternative-treatment group. Of course, this approach requires both a detailed knowledge of the evolution of the structural model over time and a large reservoir of relevant time-dependent data. It also assumes the absence of unmeasured confounders.

A first remark can conclude this section. In many cases, the mechanisms active in social sciences are not measured at the exact time of occurrence. Their measurements are given according to an aggregation over some period of time. It is then impossible, for instance, to indicate the times $t_{i}$ 's mentioned in the above equations. Therefore an adequate modeling becomes difficult or impossible. In particular, due to possible time-aggregation of causes and outcomes, feedback effects, as illustrated by equations (2) and (3), cannot be detected.

A second remark is that most mechanisms in social sciences are not instantaneous but require a period of time. Equations (4) to (10) suggest situations where this time-period characteristic creates substantial modeling difficulties. In some cases, it would also be necessary to take into account the time-pattern of the cause or of the effect over the period. For example, does 
a tax-increase on cigarettes lead to a permanent or to a temporary reduction in smoking ?

A third remark is that mechanisms themselves may change over time, leading not only to different parameters but also to different conditioning structures. For example, the determinants of fertility have drastically changed in Europe since the nineteenth century.

\section{Conclusions}

As recalled at the beginning of this paper, all of our experience of the world happens in time. Indeed, we cannot even imagine empty time (NewtonSmith, 1986). While we have not discussed the personal, inner, dimensions of time, the social sciences are nevertheless concerned with life events of individuals and/or groups that happen in time. There is therefore a 'historical' dimension of social phenomena that cannot prescind from a temporal dimension.

From a methodological perspective, the question arises how to take time into account and how to incorporate it into modeling practices. Sections 3 and 4 have surveyed the use of time in two social sciences taken as examples, demography and economics respectively. Section 5 has presented a general modeling framework, structural causal modeling (SCM), in which the core of the statistical machinery is geared towards modeling explanatory mechanisms for the phenomenon under investigation. This methodology is by design meant to be applicable across different social science domains. The way in which time, or rather information about the temporal order of variables, can be incorporated into SCM prompts the following reflection.

Analytic approaches to causality tend to put the emphasis on 'cause' and 'effect' as isolated factors among which a temporal order has to be established. This is a valid empirical question, especially in cases where there is no obvious ordering of the variables. But, in the light of how time can be used in structural models, our suggestion is to think of the relation between cause and effect more in terms of a process, of which we try to understand the key points, moments, actors, etc. This is the main idea behind SCM as presented in Section 5, in which 'structure' has a meaning that goes beyond 'statistical' structure, and that instead also has to do with 'explanation' or with 'explanatory mechanisms'. In turn, explanatory mechanisms are those

for which it is possible to offer a causal ordering of the variables that takes 
time into account. In other words, the causal structure is more important than a mere temporal ordering of the variables. This is because, with due exceptions in which the temporal order of observations corresponds to the real temporal ordering of causes and outcomes, plausibility of a causal structure will contribute more to explaining a phenomenon than the sole temporal ordering of variables as observed.

These considerations have lead us to discuss the role of time for the purpose of causal explanation in the context of social science research. Temporal order is important for modeling practices but it is not essential. Temporal information is very useful to have and should be wisely used when available. Yet, temporal information is useful only to the extent that it is placed in a correct causal structure, and thus further corroborating the mechanism explaining the phenomenon under consideration. Finally, despite the fact that the causal ordering of variables is more relevant for explanatory purposes than the temporal order, the former should nevertheless take into account the time-patterns of causes and effects, as these are often episodes rather than events. Most of our behaviors actually extend over a period of time, be they causes or effects. For this reason in particular, it is time to put time at the core of our causal models. 


\section{References}

Aalen O., Røysland K., Gran J., Ledergerber B. (2012). Causality, mediation and time: a dynamic viewpoint, Journal of the Royal Statistical Society, series A, 175, Part 4, 831-861.

Aloud M. , Tsang E., Olsen R. and Dupuis A. (2012). A directional change events approach for studying financial time series, Economics, 36, (online).

Bellotti E., Guadalupi L. And Conaldi G. (2016). Comparing fields of research: multilevel networks of research collaboration in Italian academia, chap.9 in E. Lazega and T. Snijders (Eds.): Multilevel network analysis for the social sciences, Springer, 213-244.

Bergmann W. (1992). The problem of time in sociology: an overview of the literature on the state of theory and research on the 'sociology of time', 1900-82, Time \& Society, 1(1), 81-134.

Bernardi L., Huinink J. and Settersten R.A. (2019). The life course cube: a tool for studying lives, Advances in Life Course Research, 41, https://doi.org/10.1016/j.alcr.2018.11.004

BLALOCK H.M. (1968). The measurement problem: a gap between the languages of theory and research. Chap. 1 in H.M. Blalock and A. B. Blalock (Eds.): Methodology in Social Research, McGraw-Hill, New York, 5-27.

Blossfeld H.-P. (2009). Causation as a generative process, chap. 5 in H. Engelhardt, H.-P. Kohler and A. Fürnkranz-Prskawetz, (Eds.), Causal analysis in population studies, Springer, 83-109.

Buehner M.J. (2014). Time and causality: editorial, Frontiers in Psychology, doi: 10.3389/ fpsyg. 2014.00228.

Courgeau D. (2007). Multilevel Synthesis. From the Group to the Individual, Springer.

Courgeau D. (2018). Do different approaches in population science lead to divergent or convergent models?, Chap. 2 in G. Ritschard and 
M. Studer (Eds.): Sequence analysis and related approaches, Springer, $15-33$.

Cox D.R. (1992), Causality: some statistical aspects, Journal of the Royal Statistical Society, Series A, 155(2), 291-301.

Davidson R. (2013). Time and Causality, Annals of Economics and Statistics, 109/110, 7-22.

Florens J.-P., Fougère D. And Mouchart M. (2008). Duration Models and Point Processes, chap.17 in L. Mátyás and P. Sevestre (Eds.): The Econometrics of Panel Data, Springer, 547-601.

Florens J.-P., Mouchart M. And Rolin J.-M. (1990). Elements of Bayesian Statistics, New York: Marcel Dekker.

Fosen J., Ferkingstad E., Borgan Ø., Aalen O. (2006), Dynamic path analysis- a new approach to analyzing time-dependent covariates, Lifetime Data Analysis, 12(2), 143-167.

Gerard H. (2006). Theory building in demography. Chap. 129 in G. Caselli, J. Vallin and G. Wunsch (Eds.):Demography Analysis and Synthesis. A treatise in population studies, Vol. 4, Elsevier Academic Press, San Diego, 647-659.

Gillies D. (2011). The Russo-Williamson thesis and the question of whether smoking causes heart disease, chap. 6 in P. Mackay Illari, F. Russo and J.Williamson (eds.) Causality in the Sciences, Oxford University Press, 110-125.

Glennan S. And Illari P. (2017). Varieties of Mechanisms, Chap. 7 in S. Glennan and P. Illari (Eds.): The Routledge Handbook of Mechanisms and Mechanical Philosophy, Routledge, 91-103.

Graunt J. (1662). Natural and Political Observations Made upon the Bills of Mortality, London.

HARKNESS T. (2020). John Graunt at 400. Fighting disease with numbers, Significance, 17(4), 20-25.

Hassani, H., Huang, X. And Ghodsi, M. (2018). Big Data and Causality, Annals of Data Science, 5(2), 133-156. 
Hicks J. (1980). Causality in economics, Australian National University Press, Canberra.

Illari P. And Russo F. (2014) Causality: Philosophical Theory Meets Scientific Practice. Oxford University Press.

Kelly J. And McGrath J.E. (1988).On Time and Method, Sage.

Kruschwitz L.. And Loeffler A. (2005). Discounted Cash Flow: A Theory of the Valuation of Firms, John Wiley \& Sons Inc.

LEURIDAN B. AND LODEWyCKX T. (2019) Causality and Time: an introductory typology, chap. 2 in S.Kleinberg (Ed.): Time and causality across the Sciences, Cambridge University Press, 14-36.

LitTLE D. ( 2011), Causal mechanisms in the social realm , chap. 13 in P. McKay Illari, F. Russo, and J. Williamson (Eds.): Causality in the Sciences, Oxford University Press, 273-295.

Matsuo H. And Matthijs K. (2018). The Role of Secularization on Marriage and Conception Seasonality Patterns: A Study of Antwerp (Flanders, Belgium) in the Second Half of the Nineteenth and the Early Twentieth Century, Journal of Family History, 43(4), 335-356.

Mikolai J. AND Kulu H. (2019) Union dissolution and housing trajectories in Britain, Demographic Research, 41/7, 161-196.

Mouchart M., Russo F. And Wunsch G. (2010). Inferring causal relations by modelling structures, Statistica, LXX(4), 411-432.

Muller U. A., Dacorogna M. M., Dave R. D., Pictet O. V., Olsen R. B. AND WARD, J.R. (1993, reprinted in 1995). Fractals and Intrinsic Time - a Challenge to Econometricians, Working Papers 1993-08-16, Olsen and Associates.

Newton-Smith W.H. (1986). Space, time and space-time: a philosopher's view, chap. 3 in R. Flood and M. Lockwood (Eds.): The Nature of Time, Blackwell, 22-35.

Oksuzyan, A., Petersen I., Stovring H., Bingley P., Vaupel J. W. And Christensen K. (2009). The male- female health-survival 
paradox: a survey and register study of the impact of sex-specific selection and information bias, Annals of Epidemiology, 19(7), 504-511.

PeARL J. (2000). Causality. Models, Reasoning, and Inference, Cambridge University Press, Cambridge, revised and enlarged in 2009.

Price H. (1996) Time's Arrow and Archimede's Point, Oxford University Press.

Razzaque A., Carmichael G.A. And Streatfield P.K. (2009). Adult Mortality in Matlab, Bangladesh: Levels, trends, socio-demographic differentials and causes of death, Asian Population Studies, 5(1), 85100.

Reichenbach H. (1956). The direction of time. Berkeley: University of California Press.

REISS J. (2006) Time series, nonsense correlations and the principle of the common cause, in F. Russo and J. Williamson (Eds.): Causality and Probability in the Sciences. College Publications, 179-196.

Rovelli C. (2018). The Order of Time, Penguin.

Russo F. (2009) Causality and Causal Modelling in the Social Sciences, Springer.

Russo F., Wunsch G. And Mouchart M. (2019). Causality in the social sciences: A structural modelling framework, Quality \& Quantity, 53(5), 2575-2588, doi 10.1007/s11135-019-00872-y.

RYDER N.B. (1965). The cohort as a concept in the study of social change, American Sociological Review, 30(6), 843-861.

SALmon W.C. (1994), Causality without counterfactuals, Philosophy of Science 61(2), 297-312.

Stalk, G. JR., And Thomas M. (1990). Competing Against Time: How Time-Based Competition is Reshaping Global Markets, Simon \& Shuster.

Suppes, P. (1970). A probabilistic theory of causality. Amsterdam: North Holland. 
Toulemon L. (2006). La fécondité est-elle encore naturelle ? Application au retard des naissances et à son influence sur la descendance finale, in M. Loriaux and E. Vilquin (Eds.):Entre nature et culture: quelle(s) démographie(s) ?, Chaire Quetelet 2002, Academia-Bruylant, Louvain-la-Neuve, 15-42.

VANDESCHRICK C. (2001). The Lexis diagram, a misnomer, Demographic Research, 4(3), 97-124.

VAn Tienoven T.P. (2019). A multitude of natural, social and individual time, Time \& Society, 28(3), 971-994.

Willekens F. ( 2005). Biographic Forecasting: Bridging the MicroMacro Gap in Population Forecasting, New Zealand Population Review, 31(1), 77-124.

Wunsch G. And Gourbin C. (2018). Mortality, morbidity and health in developed societies: a review of data sources, Genus, $\mathbf{7 4}(2)$. DOI: $10.1186 / \mathrm{s} 41118-018-0027-9$

Wunsch G., Mouchart M. and Russo F. (2018). Causal Attribution in Block-Recursive Social Systems - A structural modeling perspective, Methodological Innovations,11(1), 1-11.

Wunsch G., Russo F. And Mouchart M. (2010). Do we necessarily need longitudinal data to infer causal relations?, Bulletin de Méthodologie Sociologique, 106(01) 1-14.

Wunsch G. And Termote M. (1978). Introduction to Demographic Analysis. Principles and Methods, Plenum Press, New York. 


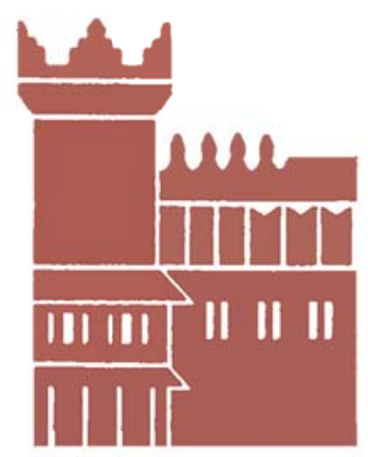

Alma Mater Studiorum - Università di Bologna DEPARTMENT OF ECONOMICS

Strada Maggiore 45

40125 Bologna - Italy

Tel. +39051 2092604

Fax +390512092664

http://www.dse.unibo.it 